\title{
Deriving Vegetation Structure in Ecological Applications from LiDAR Imagery
}

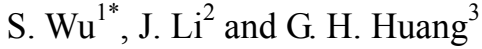 \\ ${ }^{1}$ Environmental Systems Engineering, University of Regina, Regina, SK, S4S 0A2, Canada \\ ${ }^{2}$ Department of Geography, University of Waterloo, Waterloo, ON N2L 3G1, Canada \\ ${ }^{3}$ Faculty of Engineering, University of Regina, Regina, SK, S4S 0A2, Canada
}

\begin{abstract}
Recent advances in remote sensing have presented significant potential to help improve ecological management. This paper presents an overview of Light Detection and Ranging (LiDAR) technology and its applications to derivation of vegetation structure in ecological studies. A review on relevant literature is provided to present the current status of the research on LiDAR applications. A preliminary case study is used to show a common procedure of LiDAR data analysis and some issues in real-world applications.
\end{abstract}

Keywords: Ecology, LiDAR, remote sensing, vegetation structure

\section{Introduction}

Ecology is the scientific study of the distribution and abundance of living organisms and how the distribution and abundance are affected by interactions between the organisms and their environment. Ecological research covers the scope from individual plants, to regional ecosystems, and the globe. A large part of ecological data for regional and global studies is associated with spatial extent. Over the past decades, remote sensing has played an important role in ecological studies as a realistic and cost-effective tool of acquiring data over large areas (Kerr and Ostrovsky, 2003). Conventional remote sensing techniques such as spaceborne sensing with Landsat $\mathrm{TM}$ and airborne photography have been widely applied in regional ecological studies for land cover mapping and biomass estimation. However, they cannot be easily applied to investigating vegetation structure as they are often restricted by the inability of vertical dimension measurement, and constrained to limited horizontal resolutions of their imaging sensors.

In recent years, some fine spatial resolution remote sensing systems such as Light Detection and Ranging (LiDAR) have emerged. Airborne LiDAR systems have been successfully applied to ecological applications for detailed examination of structural attributes of natural systems. (Van der Meer et al., 2002). LiDAR, in ecological studies, presents its advantage in performing topographic elevation measurements of a surface in the form of positional $\mathrm{X}, \mathrm{Y}, \mathrm{Z}$ coordinates at pre-defined intervals. The GIS data obtained from LiDAR can provide highly detailed digital surface models with vertical dimension. LiDAR imagery products are mainly being used in topographic mapping, vegetation structure measurements, and

\footnotetext{
* Corresponding author: wusimon@env.uregina.ca
}

deriving biophysical characteristics of plant communities.

Vegetation refers to the ground cover provided by plants, and is, by far, the most abundant biotic element of the biosphere. A primary characteristic of vegetation is its three-dimensional structure, sometimes referred to as its physicognomy, or architecture. Obviously, a forest has a very different structure than a desert or a backyard lawn. Vegetation ecologists discriminate structure at much more detailed levels than this, but the principle is the same. Thus, different types of forests can have very different structures; tropical rainforests are very different from boreal conifer forests, both of which differ from temperate deciduous forests. The applications of LiDAR technology to assisting with determination of vegetation structure are reviewed in this work with a demonstration of a case study.

\section{LiDAR Remote Sensing}

Light Detection and Ranging (LiDAR), as a remote sensing system, is used to collect topographic data using laser pulses. It is similar to Radio Detection and Ranging (RADAR) technology, which uses radio waves instead of light, and the range to an object is determined by measuring the time delay between transmission of a pulse and detection of the reflected signal. From 5,000 to 50,000 laser pulses in a scanning array can be emitted per second from an airborne LiDAR sensor. The most common scanning arrays go back and forth sideways relative to the points measured on the ground. The average point spacing in the cross-flight direction is determined by scan angle and flying height. The flying height and the airspeed determine the average point spacing in the inflight direction. In a common airborne LiDAR mission, a high precision Global Position System (GPS) and an Inertial Measurement Unit (IMU) are used to determine the location of the aircraft and measure the attitude so that the ground location of 
the return pulse could be accurately determined. The LiDAR sensor measures the scan angle of the laser pulses. Combined with the IMU data, this establishes the angular orientation of each laser pulse. The LiDAR sensor measures the time needed for each emitted pulse to reflect off the ground (or features thereon) and return to the sensor.

The LiDAR sensor produces a series of point measurements that consists of geographic location (horizontal $\mathrm{X}$ and Y) and height (vertical Z) of natural features. Raw LiDAR data for an area of interest are normally a very dense network of coordinate measurements with millions of points. The data are often stored in files of tabular ASCII or LAS format. A file size could be very large under a fine data resolution. The data can be processed with some specific software packages to provide input to GIS.

\section{LiDAR Applications to Vegetation Structure Derivation}

As their premier advantage in ecological studies, LiDAR sensors are capable of receiving multiple returns. The first return recorded by a LiDAR sensor is the object thing encountered by a laser pulse, normally ground, building roof, or vegetation top. The object is basically a kind of soft target in case of vegetation such as forest canopy. A portion of the laser beam on forest would most probably go through tree branches and leafs and eventually gets to the ground (Figure 1). Thus, the first sensor return normally represents the top of the vegetation feature, and last return is from earth surface. There could be one or more minor returns in between depending on the vegetation feature. The laser pulse is unable to hit the ground for vegetations of high density (e.g. certain tree species), and in this case the last return would not be present.

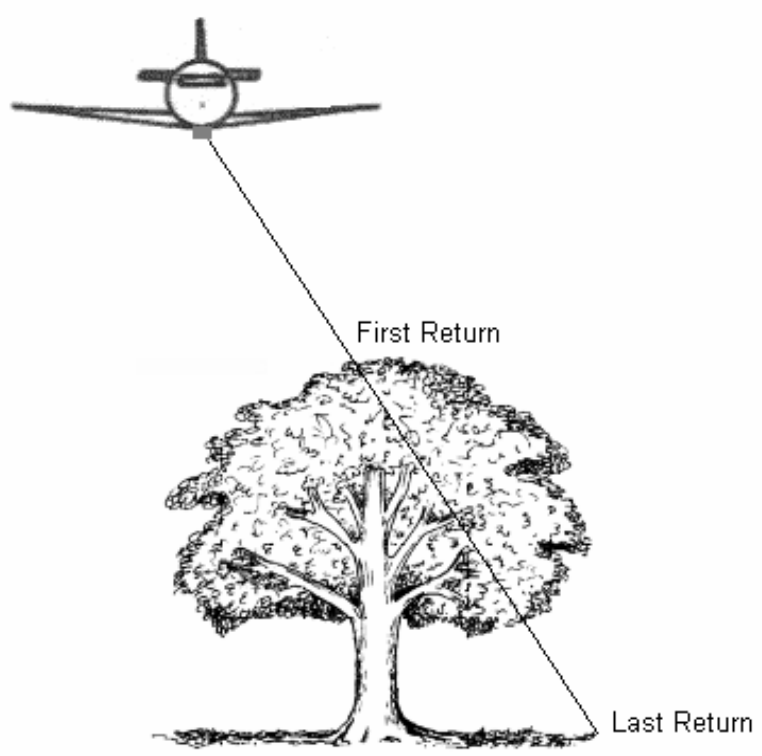

Figure 1. Multiple returns from LiDAR sensors for vegetation height.
In the work by Lefsky et al. (2001), the relationships between LiDAR-measured canopy structure and coincident field measurements of aboveground biomass at sites in the temperate deciduous, temperate coniferous, and boreal coniferous biomes were examined. A single "simplified" regression for all three sites is compared with equations derived for each site individually. The simplified equation explains $84 \%$ of variance in aboveground biomass and shows no statistically significant bias in its predictions for any individual site. Joselyn and Munro (2005) used LiDAR data to quantify current forest condition and gap distribution in a forested 141 square mile watershed is located on the west slope of the Cascade mountains. Specific derived metrics include tree height, stand density, and gap distribution. These classifications were verified based on field sample plots. The use of LiDAR data made it possible to identify and prioritize restoration project areas and to make the decision making process transparent to stakeholders. Tree heights were derived from LiDAR data of canopy heights in a work by Magnussen et al. (1999). The estimates of the expected number of 'tree top' hits were used as the probability of a LiDAR pulse hitting a tree top is governed by the number and size of tree tops. The method was demonstrated in a thinning trial with 39-year-old Douglas-fir, and only presented slightly inferior results as compared to the estimation of ground-based tree-counts. Clark et al. (2004) used a small-footprint LiDAR sensor (airborne laser scanner, ALS) to estimate sub-canopy elevation and canopy height in an evergreen tropical rain forest. A fully automated, local-minima algorithm was developed to separate LiDAR ground returns from overlying vegetation returns. We then assessed inverse distance weighted (IDW) and ordinary kriging (OK) geostatistical techniques for the interpolation of a sub-canopy digital terrain model (DTM). A digital canopy model (DCM) was calculated from the original LiDAR surface and the interpolated DTM. Individual and plot-scale heights were estimated from DCM metrics and compared to field data measured using similar spatial supports and metrics. The influence of foliage vertical distribution on vegetation gross primary production (GPP) is investigated in a study by Kotchenova et al. (2004) using LiDAR remote sensing. A new photosynthesis model has been created that combines the standard sunlit/shaded leaf separation (two-leaf) and the multiple layer approaches and uses vertical foliage profiles measured by SLICER (the Scanning LiDAR Imager of Canopies by Echo Recovery). Daily gross carbon assimilation rates calculated by this model were compared with the rates calculated by two other models, the two-leaf model and the combined two-leaf multilayer model utilizing uniform foliage profiles.

Lee et al. (2004) presented a work using small footprint LiDAR to quantify vertical forest stand structure in subtropical open forests and woodlands in Australia. It shows how small footprint LiDAR sensor data can be used to create apparent vertical profiles to describe aspects of vertical stand structure and also infer broad successional or growth stages. Such profiles were integrated with field measurements within a common reference matrix, thereby providing spatially explicit tree/crown maps in three dimensions and allowing valida- 
tion of those generated from LiDAR. Small footprint LiDAR data were also used to detect and characterize vegetation in a semi-arid sagebrush steppe environment in southeastern Idaho (Streutker and Glenn, 2006). Processing the raw data in individual flightlines maintained the high relative accuracy of the data set and allowed for the detection of sub-meter vegetation. First return LiDAR pulse data were used to both determine the ground surface as well as calculate vegetation heights. The LiDAR-determined vegetation heights are moderately well correlated to those measured in the field, although the LiDAR heights uniformly underestimate the field heights. This underestimation is believed to be due to signal threshold limits within the LiDAR sensor, producing heights corresponding to the interior of the shrub canopy rather than the top.

Andersen et al. (2004) conducted a comparative study on InSAR and high-density LiDAR data in terms of forest canopy measurements in a pacific northwest conifer forest. Their results generally indicated that both technologies can provide valuable measurements of gross canopy dimensions. In general, LiDAR elevation models acquired from high-density data more accurately represent the complex morphology of the canopy surface, while InSAR models provide a generalized, less-detailed characterization of canopy structure. Hyde et al. (2006) compared the estimates of forest structural metrics derived from remote sensing to measurements obtained in the field. Then, structural information from LiDAR, RaDAR, and passive optical sensors were statistically combined to improve accuracy of the estimates. The results of the study indicated that LiDAR is the best single sensor for estimating canopy height and biomass.

\section{LiDAR Data Analysis - A Preliminary Case Study}

The case study area is located in the Lake Qihai watershed. The LiDAR remote sensing dataset covers a mountain area of about $10 \mathrm{~km}^{2}$ below the altitude of 2,500 m where vegetation is dominated by semi-humid evergreen broadleaved forest. The forest is mainly composed of evergreen oaks and chestnuts along with some other trees (Figure 2). The locations of trees are obtained from GPS recording of plot corners and a laser distance rangefinder. A polygon layer exactly covering the $10 \mathrm{~km}^{2}$ area was created in ArcInfo GIS as the reference matrix. The LiDAR dataset as an XYZ plot was added to the layer with the first returns representing tree heights. Canopy height seems a straightforward measurement, and could be calculated by subtracting the elevations of the first and last returns of LiDAR. As a matter of fact, the height of the first LiDAR return is often lower than actual the tree top due to low density at the very top of trees. A correction model was thus developed based on the data from field measurements, and applied to the calculation of canopy heights. Figure 3 shows the LiDAR GIS map of the first returns (digital canopy model) for one part of the area. The results were analyzed and compared with the imagery from field airborne 1:20,000 photography. The consistency of coordinate registration between the two measurements was first examined and corrections were made to ensure the height $(Z)$ data are given on the same basis. The comparison indicated high correlation between the two sets of data for the area. A typical distribution of laser pulse strength in vertical dimension (tree height) is demonstrated in Figure 4.

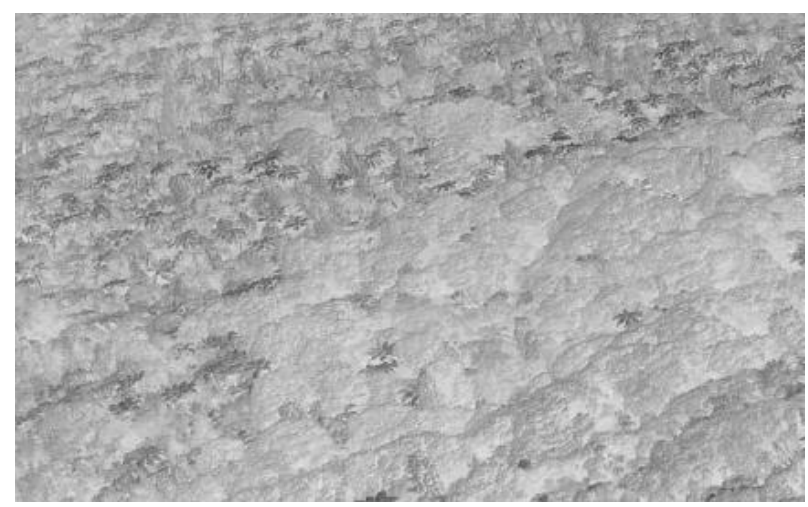

Figure 2. Forest in the study area.

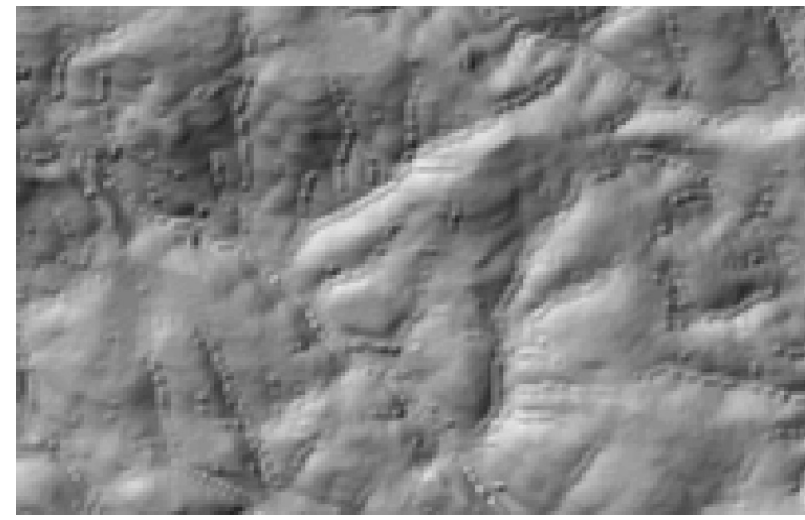

Figure 3. Digital canopy map (hillshade) with LiDAR first return.

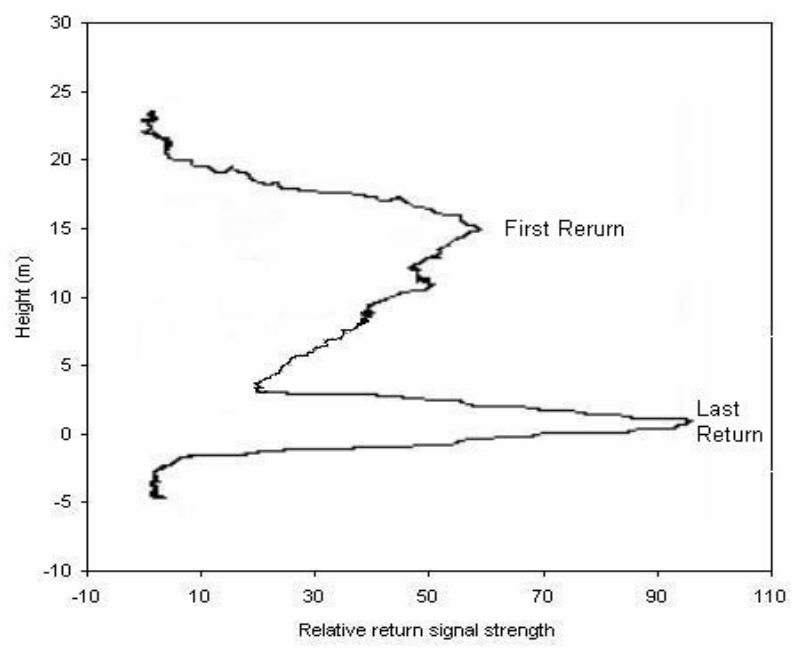

Figure 4. Vertical distribution of LiDAR returns with tree height. 


\section{Concluding Remarks}

Information about vegetation canopy structure can be derived from multiple return or full return LiDAR sensors, in addition to landscape topography. For example, an estimate of vegetation height can be obtained from the first return (vegetation top) and last return (ground surface). As well, using more sophisticated models, vegetation biomass distribution can be obtained from the profile of multiple returns. This paper has reviewed some published works in examination of vegetation structure with LiDAR data along with a preliminary case presentation.

LiDAR remote sensing has provided us with a unique tool to study vertical structure of vegetation cover on earth surface. The advantage over passive optical remote sensing systems has not been widely taken due basically to the high cost of commercial airborne LiDAR products. LiDAR data available from government agencies are very limited. Spaceborne LiDAR systems are still yet to be implemented while much desired by ecologists. Anyhow, substantial efforts and progress have been made by researchers to expand the scope of ecological applications of LiDAR with the rapid advancement of LiDAR sensors. Along with the applications of multiple return LiDAR sensors, greatly improved image processing techniques and data storage capability in GIS have made it possible to handle the ecological information obtained from LiDAR systems.

\section{References}

Andersen, H.E., McGaughey, R.J., Carson, W.W., Reutebuch, S.E., Mercer, B. and Allan, J. (2004). A comparison of forest canopy models derived from LiDAR and INSAR data in a Pacific
Northwest conifer forest. Int. Arch. Photogramm. Remote Sens., 34-3/W13, 211-217.

Clark, M.L., Clark, D.B. and Roberts, D.A. (2004). Small-footprint LiDAR estimation of sub-canopy elevation and tree height in a tropical rain forest landscape. Remote Sens. Environ., 9, 68-89.

Hyde, P., Dubayah, R., Walker, W., Blair, J.B., Hofton, M. and Hunsaker, C. (2006). Mapping forest structure for wildlife habitat analysis using multi-sensor (LiDAR, SAR/InSAR, ETM+, Quickbird) synergy. Remote Sens. Environ., 102, 63-73.

Joselyn, M. and Munro, D. (2005). Deriving forest structure from LiDAR data for a large watershed, 2005 ESRI International User Conference Proceedings, ESRI.

Kerr, J.T. and Ostrovsky, M. (2003). From space to species: Ecological applications for remote sensing. Trends Ecol. Evol., 18, 299-305.

Kotchenova, S.Y., Song, X., Shabanov, N.V., Potter, C.S., Knyazikhin, Y. and Myneni, R.B. (2004). LiDAR remote sensing for modeling gross primary production of deciduous forests. Remote Sens. Environ., 92, 158-172.

Lee, A., Lucas, R. and Brack, C. (2004). Quantifying vertical forest stand structure using small footprint LiDAR to assess potential stand dynamics. Int. Arch. Photogramm., Remote Sens. Spatial Inf. Sci., XXXVI-8/W2, 213-217.

Lefsky, M.A., Cohen, W.B., Harding, D.J., Parker, G.G., Acker, S.A. and Gower, S.T. (2001). LiDAR remote sensing of aboveground biomass in three biomes. Int. Arch. Photogramm. Remote Sens., XXXIV-3/W4, 150-160.

Magnussen, S., Gougeon, F.A., Leckie, D. and Wulder, M. (1999). Predicting tree heights from a combination of LiDAR canopy heights and digital stem counts, in Procs. of Remote Sensing and Forest Monitoring Conference, Rogow, Poland.

Streutker, D. and Glenn, N. (2006). LiDAR measurement of sagebrush steppe vegetation heights. Remote Sens. Environ., 102, 135-145.

Van der Meer, F., Schmidt, K.S., Bakker, W. and Bijker, W. (2002). New environmental remote sensing systems, in A. Skidmore (Ed.), Environmental Modelling with GIS and Remote Sensing, Taylor \& Francis, London, pp. 26-51. 